\title{
Interaction of optical solitons with a forward Raman pump wave
}

\author{
Sien Chi and Senfar Wen \\ Institute of Electro-Optical Engineering, National Chiao Tung University, Hsinchu, Taiwan, China
}

Received June 1, 1988; accepted October 14, 1988

\begin{abstract}
The interaction between optical solitons and a forward Raman pump wave in fibers is investigated. It is found that when their relative velocity is small and the soliton pulse width is short, the soliton frequency is shifted by the interaction. Near zero relative velocity the pulse shape is distorted, in addition to having a frequency shift.
\end{abstract}

The optical soliton has been proposed as an information carrier in fiber communication systems. ${ }^{1} \mathrm{Be}-$ cause of the fiber loss, the soliton disperses as it propagates along the fiber. Use of periodically bidirectional Raman pumps to compensate for the loss has been suggested. ${ }^{2,3}$ Numerical calculations showed that the soliton can preserve its pulse shape for thousands of kilometers with this method. The experiment also showed that the dispersion of the soliton due to loss can be reduced by a backward Raman pump. ${ }^{4}$ In previous theoretical research, Raman pump waves were assumed to be either uniformly depleted ${ }^{2}$ or undepleted. ${ }^{3}$ With the assumption of uniform depletion, the pump wave is assumed to be a cw wave along the fiber. This is valid if the relative group velocity between the pump wave and the soliton is sufficiently large. The relative group velocity between a backward pump wave and the soliton is large enough, but this is not in general true for a forward pump wave. In this Letter we show the effects caused by a small relative group velocity between a forward pump wave and the soliton.

The soliton and a forward pump wave in a fiber satisfy the following coupled dimensionless wave equation ${ }^{2}$ :

$$
\begin{aligned}
& \begin{aligned}
i \frac{\partial q_{s}}{\partial \xi}+\frac{1}{2} \alpha_{s} \frac{\partial^{2} q_{s}}{\partial \tau^{2}}+\eta_{s}\left(\left|q_{s}\right|^{2}\right. & \left.+2\left|q_{p}\right|^{2}\right) q_{s} \\
& =-i \Gamma_{s} q_{s}+i G\left|q_{p}\right|^{2} q_{s},
\end{aligned} \\
& \begin{aligned}
i\left(\frac{\partial q_{p}}{\partial \xi}-V \frac{\partial q_{p}}{\partial \tau}\right)-\frac{1}{2} \alpha_{p} \frac{\partial^{2} q_{p}}{\partial \tau^{2}}+\eta_{p}\left(\left|q_{p}\right|^{2}+2\left|q_{s}\right|^{2}\right) q_{p} \\
=
\end{aligned} \\
& =-i \Gamma_{p} q_{p}-i \frac{\lambda_{s}}{\lambda_{f}} G\left|q_{s}\right|^{2} q_{p},
\end{aligned}
$$

where the nonlinear coupling of the soliton $\left(q_{s}\right)$ and the forward pump wave $\left(q_{p}\right)$ are due to the Kerr effect and Raman scattering. The Kerr effect causes the phases of $q_{s}$ and $q_{p}$ to be mutually modulated. The variables and the coefficients in the above equations are normalized in the following way:

$$
\begin{gathered}
\xi=X^{-1} z, \quad \tau=T^{-1}\left(t-k_{s}^{\prime} z\right), \quad q_{s}=Q^{-1} \phi_{s}, \\
q_{p}=Q^{-1} \phi_{p}
\end{gathered}
$$

and

$$
\begin{gathered}
\alpha_{s}=-k_{s}^{\prime \prime} X T^{-2}, \quad \alpha_{p}=k_{p}{ }^{\prime \prime} X T^{-2}, \\
\eta_{s}=\frac{\pi n_{2}}{\lambda_{s}} Q^{2} X, \quad \eta_{p}=\frac{\pi n_{2}}{\lambda_{f}} Q^{2} X, \\
\Gamma_{s}=\gamma_{s} X, \quad \Gamma_{p}=\gamma_{p} X, \quad G=\frac{n_{0}}{4} \sqrt{\epsilon_{0} / \mu_{0}} g X Q^{2}, \\
V=\left(k_{p}{ }^{\prime}-k_{s}{ }^{\prime}\right) X T^{-1} .
\end{gathered}
$$

In Eqs. (1)-(3), $\phi_{s}$ and $\phi_{p}$ are the electric-field envelopes of the soliton and the forward pump wave; $\lambda_{s}$ and $\lambda_{p}$ are the wavelengths of the soliton and the pump wave; $t$ and $z$ are the time and propagation distance along the fiber; $T, X$, and $Q$ are the time, distance, and electric-field scale factors; $k_{s}{ }^{\prime}, k_{s}{ }^{\prime \prime}$ and $k_{p}{ }^{\prime}, k_{p}{ }^{\prime \prime}$ are the derivatives of the propagation constant $k$ with respect to the angular frequencies at $\omega_{s}$ and $\omega_{p}$, respectively, where $\omega_{s}$ and $\omega_{p}$ are the angular frequencies of the soliton and the pump wave, the first derivative is reciprocal group velocity, and the second derivative is proportional to group dispersion; $\gamma_{s}$ and $\gamma_{p}$ are the loss coefficients of the fiber at $\lambda_{s}$ and $\lambda_{p} ; n_{2}$ is the Kerr coefficient and equal to $1.22 \times 10^{-22}(\mathrm{~V} / \mathrm{m})^{-2}$ for fused silica; $g$ is the Raman gain coefficient, which is maximum when the frequency difference between the pump wave and the carrier wave of the soliton is 440 $\mathrm{cm}^{-1} .5$ The maximum gain $g=9.75 \times 10^{-14} / \lambda_{p} \mathrm{~m} / \mathrm{W}$, where $\lambda_{p}$ is in micrometers. In the following we take $X=10 \mathrm{~km}, Q=4.55 \times 10^{5} \mathrm{~V} / \mathrm{m}$, and let $T$ be varied $(T$ is in picoseconds). The given electric-field scale corresponds to $20 \mathrm{~mW}$ for a $50-\mu \mathrm{m}^{2}$ fiber cross section.

The soliton wavelength is in the negative-dispersion regime $\left(k_{s}{ }^{\prime \prime}<0\right)$. We assume, however, that the pump wave is in the positive-dispersion regime $\left(k_{p}^{\prime \prime}>0\right)$. Because the reciprocal group velocity is minimum at zero dispersion, the relative reciprocal group velocity $\Delta k^{\prime}\left(=k_{p}{ }^{\prime}-k_{s}{ }^{\prime}\right)$ may be small. Using the dispersion relation in Ref. 6 for fused silica and a $440-\mathrm{cm}^{-1}$ frequency difference between $\lambda_{s}$ and $\lambda_{p}$, we plot $\Delta k^{\prime}$ in Fig. 1. There, the zero-dispersion wavelength is assumed to be at $1.273 \mu \mathrm{m}$, and then $\Delta k^{\prime}=0$ at $\lambda_{s}=$ $1.30885 \mu \mathrm{m}$. This wavelength is denoted by $\lambda_{s 0}$.

We first consider the interaction of the soliton and a forward pump wave at $\lambda_{s}=1.3 \mu \mathrm{m}$ and $\lambda_{p}=1.23 \mu \mathrm{m}$. 


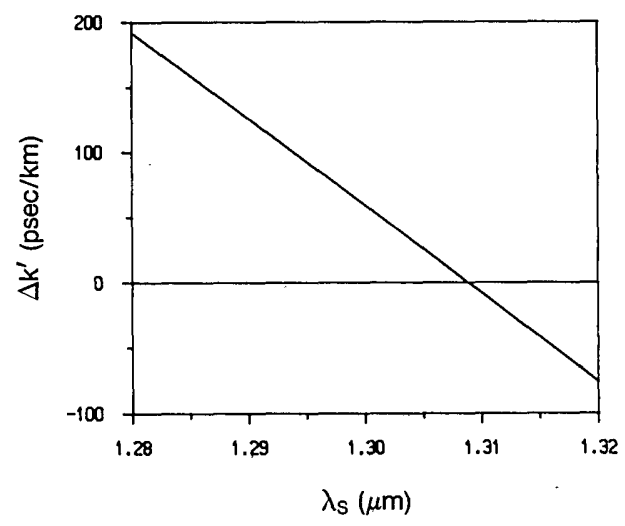

Fig. 1. Relative reciprocal group velocity $\Delta k^{\prime}$ between the Raman pump wave and the carrier wave of the soliton with a frequency difference of $440 \mathrm{~cm}^{-1}$.

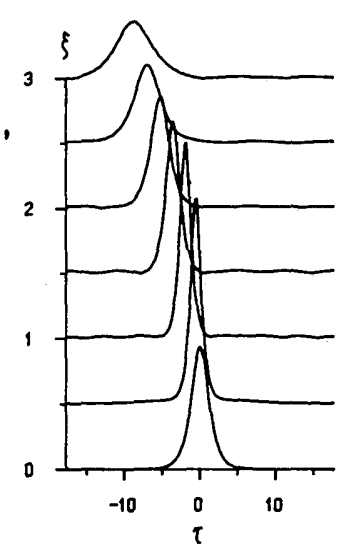

(a)

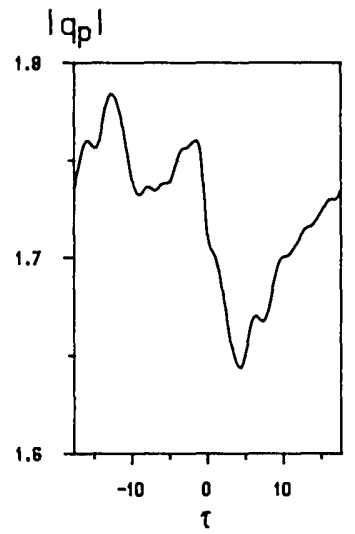

(b)
Fig. 2. (a) Envelope evolution of the soliton amplified by the forward pump wave. The soliton has a wavelength of 1.3 $\mu \mathrm{m}$ and an initial amplitude of 6.25. The pump wave has a wavelength of $1.23 \mu \mathrm{m}$ and an initial amplitude of 3 . The time scale $T=1 \mathrm{psec}$, and the relative velocity $V=590$. (b) Envelope of the forward pump wave at $\xi=0.5$.

In this case, $k_{s}^{\prime \prime}=-2.37 \mathrm{psec}^{2} / \mathrm{km}, k_{p}{ }^{\prime \prime}=3.62 \mathrm{psec}^{2} /$ $\mathrm{km}$, and $\Delta k^{\prime}=59 \mathrm{psec} / \mathrm{km}$. The loss coefficients are assumed to be $\gamma_{s}=0.3 \mathrm{~dB} / \mathrm{km}$ and $\gamma_{p}=0.5 \mathrm{~dB} / \mathrm{km}$. The initial conditions are taken to be

$$
q_{s}(\tau, \xi=0)=\sqrt{\alpha_{s} / \eta_{s}} \operatorname{sech}(\tau), \quad q_{p}(\tau, \xi=0)=q_{p 0} .
$$

Initially, $q_{s}$ is the fundamental soliton with amplitude $\sqrt{\alpha_{s} / \eta_{s}}$ and $q_{p}$ is the cw wave with amplitude $q_{p 0}$. The pulse width (FWHM) of the sech pulse is $1.76 T$ (psec). Note that, from Eqs. (3) and (4), the pulse amplitude is inversely proportional to the time scale $T$, which is proportional to the pulse width. It is shown below that the effects caused by the interaction between them is increased as $T$ is decreased. Equations (1) are numerically solved by the propagating-beam $\operatorname{method}^{7}$ with periodic boundary conditions. We take $\tau=$ -17.6 to 17.6 , i.e., what we consider is an infinite pulse train with 20 pulse widths between neighboring solitons.
The envelope evolution of the soliton is shown in Fig. 2(a) for $T=1 \mathrm{psec}(V=590)$ and $q_{p 0}=3$. The envelope of the forward pump wave at $\xi=0.5$ is shown in Fig. 2(b). In Fig. 2(b), the pump wave is depleted by the soliton, in addition to the decay due to fiber loss. The depletion makes the envelope of the pump wave fluctuate and leads to the cross-phase modulation $^{8,9}$ between the pump wave and the soliton. We have found that the cross-phase modulation shifts the carrier frequency of the soliton. It is seen in Fig. 2(a) that the soliton is at first amplified and finally decays owing to pump depletion and fiber loss. Furthermore, the soliton propagates to the negative direction of the $\tau$ frame, which is the moving frame with the velocity of the initial soliton; this shows that the velocity of the soliton is changed. The velocity change is due to the frequency shift of the soliton. $\delta f$ can be calculated by ${ }^{3}$

$$
\delta f=\frac{-1}{2 \pi T} \frac{\operatorname{Im} \int q_{s}{ }^{*} \frac{\partial}{\partial \tau} q_{s} \mathrm{~d} \tau}{\int q_{s}{ }^{*} q_{s} \mathrm{~d} \tau} .
$$

Figure 3 shows the frequency shift $\delta f$ along the fiber. At approximately $\xi=2, \delta f$ reaches a steady-state value. After $\xi=2$, the contribution of the forward pump wave can be neglected. We define the steady-state value to be $\delta f_{s}$. In Fig. $3, \delta f_{s}=22.8 \mathrm{GHz}$. $\delta f_{s}$ for initial

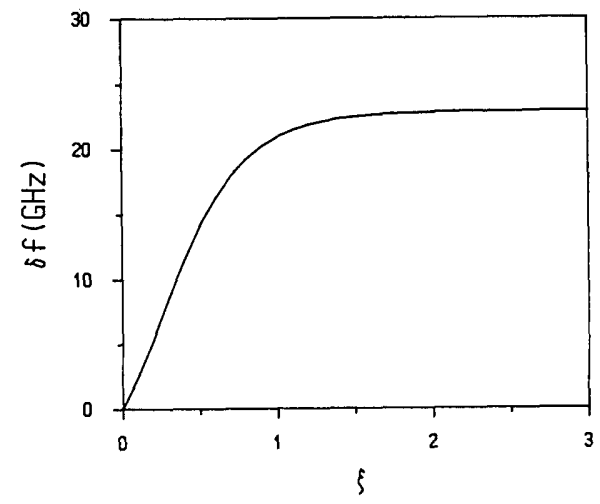

Fig. 3. Frequency shift $\delta f$ of the soliton along the fiber shown in Fig. 2(a).

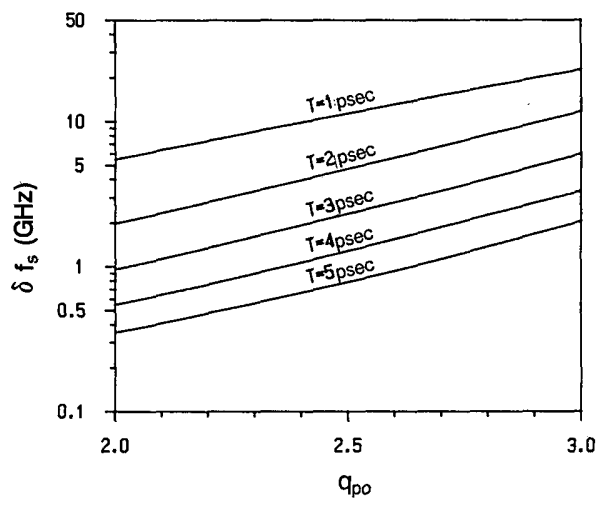

Fig. 4. Steady-state frequency shift $\delta f_{s}$ of the soliton with a wavelength of $1.3 \mu \mathrm{m}$ amplified by a forward Raman pump wave with initial amplitude $q_{p 0}$ for several time scales. 


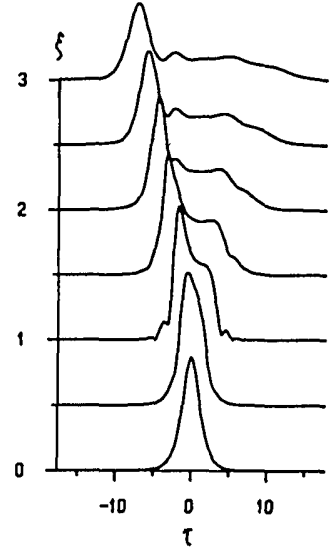

(a)

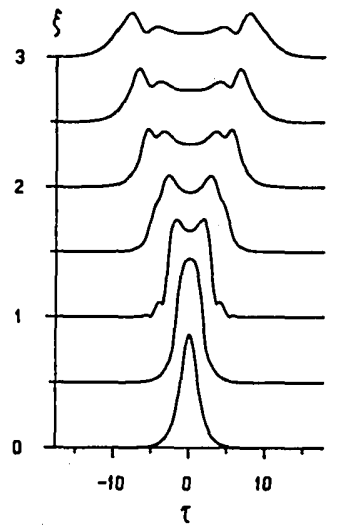

(b)
Fig. 5. Envelope evolution of the soliton with time scale $T$ $=5 \mathrm{psec}$ and $\mathrm{a}$ wavelength of (a) $1.3087 \mu \mathrm{m}$ and (b) $1.3885 \mu \mathrm{m}$ amplified by the forward pump wave with an initial amplitude of 3. The initial soliton amplitudes are (a) 1.443 and (b) 1.444

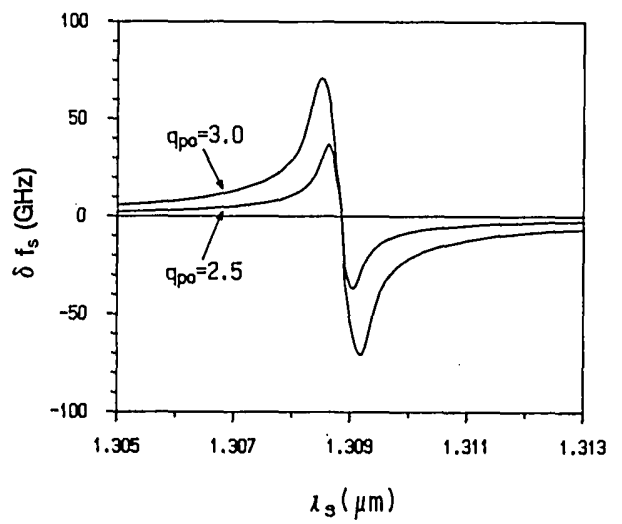

Fig. 6. Steady-state frequency shift $\delta f_{s}$ of the soliton with time scale $T=5$ psec and a wavelength near zero relative velocity wavelength $\lambda_{s 0}$ amplified by a forward Raman pump wave with initial amplitudes $q_{p 0}$ of 2.5 and 3 .

pump amplitudes varied from 2 to 3 , and several time scales with $\lambda_{s}=1.3 \mu \mathrm{m}$ are shown in Fig. 4 . From the figure we see that $\delta f_{s}$ increases with the initial pump amplitude $q_{p 0}$. For $T=1 \mathrm{psec}, \delta f_{s}$ increases exponentially with $q_{p 0}$, while, for $T=5 \mathrm{psec}, \delta f_{s}$ increases exponentially with $q_{p 0}{ }^{2}$. For $T$ between 1 and 5 psec, $\delta f_{s}$ increases exponentially with $q_{p 0}$ by a power between 1 and 2. Note that $\delta f_{s}$ increases when $T$ de- creases. This is due to higher depletion for the larger soliton power with a smaller $T$ or pulse width.

Next we study the cases around $\lambda_{s 0}$, the wavelength of the soliton at zero relative velocity. For $T=5 \mathrm{psec}$ and $q_{p 0}=3$, Fig. 5 shows the cases with $\lambda_{s}=1.3087 \mu \mathrm{m}$ and $\lambda_{s}=\lambda_{s 0} . \quad V=1.63$ for the case with $\lambda_{s}=1.3087$ $\mu \mathrm{m}$. From the figure we see that the pulse shapes distort significantly for both cases. Although the corresponding frequency spectrum also distorts greatly, there is a steady-state frequency shift. In such a case, the frequency shift calculated from Eq. (5) can be referred to the average frequency spectrum shift. For fixed $T$ and $q_{p 0}$, the steady-state frequency shift $\delta f_{s}$ for the cases with $\lambda_{s}$ around $\lambda_{s 0}$ is shown in Fig. 6. The sign of $\delta f_{s}$ is reversed from $\lambda_{s}<\lambda_{s 0}$ to $\lambda_{s}>\lambda_{s 0}$ for a different sign of relative velocity. There are extrema on both sides of $\lambda_{s 0}$. As $\lambda_{s}$ is away from the wavelength of the extremum, $\delta f_{s}$ decreases rapidly, and the corresponding pulse-shape distortion is reduced. Note that $\lambda_{s 0}$ is away from $1.3 \mu \mathrm{m}$ by only a few nanometers. This indicates that a slight source frequency shift may lead the soliton to the large pulse-shape distortion regime.

In conclusion, we have considered the interaction between the soliton and a forward pump wave. When their relative velocity is small and the soliton pulse width is short, the soliton's frequency is shifted by the interaction. Near zero relative velocity, the pulse shape is distorted, in addition to its experiencing a frequency shift. Therefore, to avoid frequency shift and pulse-shape distortion, either the pulse width cannot be too short or the soliton and pump frequencies should be properly chosen so that the relative velocity between the forward pump wave and the soliton is not too small.

\section{References}

1. A. Hasegawa and Y. Kodama, Proc. IEEE 69, 1145 (1981).

2. A. Hasegawa, Appl. Opt. 23, 3302 (1984).

3. L. F. Mollenauer, J. P. Gordon, and M. N. Islam, IEEE J. Quantum Electron. QE-22, 157 (1986).

4. L. F. Mollenauer and R. H. Stolen, Opt. Lett. 10, 229 (1985).

5. L. B. Jeunhomme, Single-Mode Fiber Optics (Dekker, New York, 1983), p. 257.

6. I. H. Malitson, J. Opt. Soc. Am. 55, 1205 (1965).

7. D. Yevick and B. Hermansson, Opt. Commun. 47, 101 (1983).

8. J. Gersten, R. Alfano, and M. Belic, Phys. Rev. A 21, 1222 (1980).

9. D. Schadt, B. Jaskorzynska, and U. Osterberg, J. Opt. Soc. Am. B 3, 1257 (1986). 